\title{
The solution of Kato's conjecture (after Auscher, Hofmann, Lacey, Mc Intosh and Tchamitchian)
}

\author{
Philippe Tchamitchian
}

\begin{abstract}
Kato's conjecture, stating that the domain of the square root of any accretive operator $L=-\operatorname{div}(A \nabla)$ with bounded measurable coefficients in $\mathbb{R}^{n}$ is the Sobolev space $H^{1}\left(\mathbb{R}^{n}\right)$, i.e. the domain of the underlying sesquilinear form, has recently been obtained by Auscher, Hofmann, Lacey, $\mathrm{M}^{\mathrm{C}}$ Intosh and the author. These notes present the result and explain the strategy of proof.
\end{abstract}

\section{Introduction}

Let $A=A(x)$ be an $n \times n$ matrix of complex bounded coefficients, defined on $\mathbb{R}^{n}$, and satisfying the ellipticity condition

$$
\left|A(x) \xi \cdot \zeta^{*}\right| \leq \Lambda|\xi||\zeta| \text { and } \lambda|\xi|^{2} \leq \operatorname{Re} A(x) \xi \cdot \xi^{*}
$$

for all $x \in \mathbb{R}^{n}, \xi, \zeta \in \mathbb{C}^{n}$. Here, $\lambda$ and $\Lambda$ are positive; also, if $u, v \in \mathbb{C}^{n}, u \cdot v$ stands for $u_{1} v_{1}+\cdots+u_{n} v_{n}, v^{*}$ is the complex conjugate of $v$, and thus $u \cdot v^{*}$ is the usual inner product in $\mathbb{C}^{n}$.

To $A$ is associated the sesquilinear accretive form

$$
J(f, g)=\int_{\mathbb{R}^{n}} A \nabla f \cdot \overline{\nabla g},
$$

whose domain is the Sobolev space $H^{1}\left(\mathbb{R}^{n}\right)$; Its accretivity allows to define the second order operator

$$
L=-\operatorname{div}(A \nabla),
$$

which is itself maximal accretive. Therefore, it has a functional calculus, and in particular a square root $\sqrt{L}$.

MSC 2000: 35J15, 47A60, 42B25.

Keywords : second order complex elliptic operators, square root problem, rough quadratic functionals, Carleson measures. 
When $A$ is real symmetric, so that $L=L^{*}$, it is elementary to check that $D(\sqrt{L})$, the domain of $\sqrt{L}$, is $H^{1}\left(\mathbb{R}^{n}\right)$ and more precisely that

$$
\|\sqrt{L} f\|_{2}^{2}=\langle L f, f\rangle \sim\|\nabla f\|^{2} .
$$

In the beginning of the 60's, motivated by the study of time-dependent evolution equations, Kato asked how, in this case, the square root $\sqrt{L}$ depends on the coefficients of $A[\mathrm{~K}]$ :

Question 1. Is the application $A \mapsto \sqrt{L}$, defined from the set of real symmetric elliptic matrices to the space of bounded operators from $H^{1}\left(\mathbb{R}^{n}\right)$ to $L^{2}\left(\mathbb{R}^{n}\right)$, analytic?

Through a standard Cauchy integral argument, it is enough to show that $D(\sqrt{L})$ is equal to $H^{1}\left(\mathbb{R}^{n}\right)$ when $A$ is complex-valued with, at least, a small imaginary part, to get a positive answer to Question 1 . This leads naturally to

Question 2 (The square root problem). In the general case, is it still true that $D(\sqrt{L})=H^{1}\left(\mathbb{R}^{n}\right)$ ?

These two questions have been answered affirmatively. First, in 2000, Auscher, Hofmann, Lewis and Tchamitchian proved the analyticity result [AHLT]; then, a few months later, Auscher, Hofmann, Lacey, $M^{\mathrm{c}}$ Intosh and Tchamitchian solved the square root problem [AHLMT]. Both proofs have the same starting point and rely on Carleson measures estimates, which are obtained by different means.

Here, we will concentrate on the proof of the strongest result, which can be precisely stated as follows.

Theorem 1. For any operator as above the domain of $\sqrt{L}$ coincides with the Sobolev space $H^{1}\left(\mathbb{R}^{n}\right)$ and

$$
\|\sqrt{L} f\|_{2} \sim\|\nabla f\|_{2}
$$

with constants only depending on $\lambda$ and $\Lambda$.

Proving this theorem amounts to show that

$$
\|\sqrt{L} f\|_{2} \leq C\|\nabla f\|_{2}
$$

for $f$ in some dense subspace of $H^{1}\left(\mathbb{R}^{n}\right)$, with $C$ depending only on $n, \lambda$ and $\Lambda$ (since, using the accretivity of the form $J$, this inequality for $L^{*}$ implies the converse inequality for $L$ ).

Further comments, references and generalisations may be found in [M83], [AT], [AHLT], [AHLMT], [AHLLMT].

\section{A few words on the abstract problem}

Actually, Kato formulated his two questions in an abstract setting, i.e. for general maximal accretive operators defined through accretive forms [K]. In 1972, McIntosh gave a negative answer to both of them [M72]. However, his counter-examples 
were not differential operators, so that the questions remained entirely open for divergence second order operators.

Here is a somewhat simplified abstract counter-example, extracted from [AT], which relies on classical harmonic analysis.

Take $H=l^{2}(\mathbb{Z})$, choose $\left(b_{j}\right)_{j \in \mathbb{Z}}$ such that its symbol $b(\theta)=\sum_{j} b_{j} e^{i j \theta}$ is a bounded function with $\|b\|_{\infty}=1$, and define two operators $D$ and $B$ by

$$
\begin{gathered}
D:\left(c_{j}\right)_{j \in \mathbb{Z}} \mapsto\left(2^{j} c_{j}\right)_{j \in \mathbb{Z},} \\
B:\left(c_{j}\right)_{j \in \mathbb{Z}} \mapsto\left(\sum_{k} c_{j-k} b_{k}\right)_{j \in \mathbb{Z} .}
\end{gathered}
$$

Then, $D$ is unbounded with domain $V$ dense in $H$, while $B$ is bounded with norm 1 .

For any $z \in \mathbb{C},|z|<1$, the form

$$
J_{z}(u, v)=\langle(I+z B) D u, D v\rangle_{H}
$$

has domain $V$ and is accretive. We define the operator

$$
L_{z}=D(I+z B) D,
$$

and consider $R_{z}=\sqrt{L_{z}}$.

Let us assume that there exists $r>0$ such that

$$
\forall u \in H \quad\left\|R_{z} u\right\|_{H} \leq C\|D u\|_{H}
$$

for some $C>0$, whenever $|z|<r$. Then the application $z \mapsto R_{z}$ is differentiable at $z=0$, and we must have

$$
\forall u \in H \quad\left\|R_{0}^{\prime} u\right\|_{H} \leq C^{\prime}\|D u\|_{H} .
$$

Starting from the identity

$$
R_{0}^{\prime} D+D R_{0}^{\prime}=D B D,
$$

one can compute $R_{0}^{\prime} u, u \in H$ : it is the convolution between $D u$ and the sequence $\left(\tilde{b}_{j}\right)_{j \in \mathbb{Z}}$ defined by

$$
\tilde{b}_{j}=\frac{2^{j}}{2^{j}+1} b_{j} .
$$

Therefore its symbol $\tilde{b}(\theta)=\sum_{j} \tilde{b}_{j} e^{i j \theta}$ is bounded if and only if the conjugate function of $b$ is itself bounded. This shows that $R_{0}^{\prime} D^{-1}$ may not be bounded, contradicting (1).

As the reader may see, this counter-example is not a differential operator. The same observation was true for the original counter-examples of $\mathrm{M}^{\mathrm{c}}$ Intosh, who consequently specified Kato's questions, giving them their present form.

Moreover, $\mathrm{M}^{\mathrm{c}}$ Intosh came to the feeling that the square root problem and the so-called Calderón conjectures were probably linked [M82]. Indeed, his counterexamples are related to the fact that some abstract commutator may not be bounded, while in the setting of differential operators in dimension one, this commutator becomes the first Calderón commutator, the boundedness of which has been obtained by Calderón in 1965 [C]. 


\section{The one-dimensional case}

The intuition of $\mathrm{M}^{\mathrm{c}}$ Intosh was correct. In their celebrated paper where they proved $L^{2}$-continuity of the Cauchy operator on Lipschitz curves, Coifman, M ${ }^{\mathrm{c}}$ Intosh and Meyer also solved the square root problem in dimension one [CMM] (and later the two problems were shown by Kenig and Meyer to be equivalent: see [KM]). They used techniques of multilinear analysis, which subsequently lead to partial results on the square root problem in several dimensions [CDM], [FJK], [M85], [J]. All these results are of perturbative nature, reflecting the fact that the underlying multilinear operators have norms with presumably exponential growth when $n \geq 2$.

Let us instead introduce some different ideas, relevant whatever the dimension is, and give a "modern" proof of the one-dimensional case.

We note $a$ for $A$, and $D$ for $\frac{1}{i} \frac{d}{d x}$. The operator $L$ writes $D a D$, and we want to show that $\|\sqrt{L} f\|_{2} \leq C\|D f\|_{2}$ for suitable $f$. There are three steps.

Step 1: reduction to a quadratic inequality. Abstract results of Yagi and $\mathrm{M}^{\mathrm{c} I n t o s h}([\mathrm{Y}]$, [M86]) show that the Littlewood-Paley quadratic functionals on $L^{2}\left(\mathbb{R}^{n}\right)$, classically defined with functions of the laplacian, may also be defined with maximal accretive operators under suitable conditions. In particular, we have

$$
\int_{0}^{+\infty}\left\|\left(I+t^{2} L\right)^{-1} t \sqrt{L} g\right\|_{2}^{2} \frac{d t}{t} \sim\|g\|_{2}^{2}
$$

for all $g \in L^{2}(\mathbb{R})$. Taking $f$ in $D(L)$ and $g=\sqrt{L} f$, inequality $(\mathrm{K})$ becomes

$$
\int_{0}^{+\infty}\left\|\left(I+t^{2} L\right)^{-1} t D a D f\right\|_{2}^{2} \frac{d t}{t} \leq C\|D f\|_{2}^{2}
$$

which (because we are in dimension one) is equivalent to

$$
\int_{0}^{+\infty}\left\|\left(I+t^{2} L\right)^{-1} t D a g\right\|_{2}^{2} \frac{d t}{t} \leq C\|g\|_{2}^{2}, \quad g \in L^{2}(\mathbb{R}) .
$$

Before going further, let us explain why we prefer to deal with quadratic functionals like in (3) instead of singular integrals, like in Kato's formula

$$
\sqrt{L}=\frac{2}{\pi} \int_{0}^{+\infty}\left(I+t^{2} L\right)^{-1} t L \frac{d t}{t} .
$$

This formula would lead to proving the $L^{2}$-continuity of the operator

$$
T=\frac{2}{\pi} \int_{0}^{+\infty}\left(I+t^{2} L\right)^{-1} t D a \frac{d t}{t}
$$

as in [CMM] and other earlier papers. Hence, the singular integral approach gives operators of the form

$$
\int_{0}^{+\infty} U_{t} \frac{d t}{t}
$$


and the quadratic functionals yield to

$$
\int_{0}^{+\infty}\left\|U_{t} \cdot\right\|_{2}^{2} \frac{d t}{t}
$$

By developing $\left\|U_{t} g\right\|_{2}^{2}=\left\langle U_{t}^{*} U_{t} g, g\right\rangle$, we see that a quadratic estimate also involves a singular integral operator, namely

$$
\int_{0}^{+\infty} U_{t}^{*} U_{t} \frac{d t}{t}
$$

The point is that in most cases each $U_{t}$ is a rough integral operator (with a rough kernel), while $U_{t}^{*} U_{t}$ is less rough, since its kernel writes

$$
U_{t}^{*} U_{t}(x, y)=\int \overline{U_{t}(z, x)} U_{t}(z, y) d z
$$

Moreover, two-sided regularity properties of $U_{t}^{*} U_{t}(x, y)$ are linked to one-sided regularity properties of $U_{t}(x, y)$, with respect to the $y$ variable only.

In the square root problem, it turns out that there is not much difference in one dimension between the two points of view. Our comment will be, however, very relevant in higher dimensions.

Step 2: reduction to a Carleson estimate. Define $\theta_{t}=\left(I+t^{2} L\right)^{-1} t D a, t>0$. For any complex-valued $a$, the resolvent operator $\left(I+t^{2} L\right)^{-1}$ has a kernel with pointwise exponential decay off the diagonal: see [AMT], where this property is shown to hold when $n=1$ or 2 . Moreover, thanks to the ellipticity of the operator $D$ (dimension one again!), this property transfers to $\theta_{t}(x, y)$, the kernel of $\theta_{t}$, and its derivatives:

$$
\begin{gathered}
\forall x, y \in \mathbb{R} \quad \forall t>0 \quad\left|\theta_{t}(x, y)\right| \leq C \frac{1}{t} e^{-\alpha \frac{|x-y|}{t}}, \\
\forall x, y \in \mathbb{R} \quad \forall t>0 \quad\left|\frac{\partial}{\partial y} \theta_{t}(x, y)\right| \leq C \frac{1}{t^{2}} e^{-\alpha \frac{|x-y|}{t}},
\end{gathered}
$$

where $\mathrm{C}$ and $\alpha$ only depend on $\lambda$ and $\Lambda$ ([AT95]). Such estimates allow to apply the following general criterion.

Theorem 2 (Christ and Journé [CJ]). For any $\left(\theta_{t}\right)_{t>0}$ fulfilling (4) and (5), the quadratic inequality

$$
\forall g \in L^{2}(\mathbb{R}) \quad \int_{0}^{+\infty}\left\|\theta_{t} g\right\|_{2}^{2} \frac{d t}{t} \leq C\|g\|_{2}^{2}
$$

is equivalent to the Carleson measure estimate

$$
\int_{0}^{|I|} \int_{I}\left|\theta_{t}(1)(x)\right|^{2} \frac{d x d t}{t} \leq C|I|,
$$

where $I$ is any interval in $\mathbb{R}$, and $|I|$ denotes its length. 
This is a T(1) theorem for quadratic functionals. Provided (4) and (5) be suitably generalised, it is valid in any dimension, with kernels polynomially decreasing off the diagonal fastly enough.

The idea behind its proof is that the operator $\theta_{t}(1) P_{t}$, where the function

$$
\theta_{t}(1)(x)=\int_{\mathbb{R}^{n}} \theta_{t}(x, z) d z
$$

acts as a pointwise multiplier, and with $\left(P_{t}\right)_{t>0}$ a smooth approximation of the identity, mimicks the operator $\theta_{t}$ itself. This is expressed by the inequality

$$
\int_{0}^{+\infty}\left\|\theta_{t} g-\theta_{t}(1) P_{t} g\right\|_{2}^{2} \frac{d t}{t} \leq C\|g\|_{2}^{2}
$$

where $\mathrm{C}$ depends on the constants in (4) and (5).

This inequality is an almost-orthogonality estimate. Define

$$
\tilde{\theta}_{t}=\theta_{t}-\theta_{t}(1) P_{t}
$$

so that $\tilde{\theta}_{t}$ fulfills (4) and (5), with in addition the cancellation property

$$
\tilde{\theta}_{t}(1)=0 \text {. }
$$

Then, expand

$$
g=\int_{0}^{+\infty} Q_{t}^{2} g \frac{d t}{t}
$$

in a standard way $\left(Q_{t}=\psi\left(-t^{2} \Delta\right)\right.$ for suitable $\left.\psi\right)$. Properties (4), (5) and (9) imply that

$$
\left\|\tilde{\theta}_{t} Q_{s}\right\|_{2,2} \leq C \min \left(\frac{t}{s}, \frac{s}{t}\right)^{\beta}
$$

for some $\beta>0$ and for all $s, t>0$, from which it is easy to deduce (8).

Now, inequality (8) shows that the quadratic inequality (6) is equivalent to

$$
\int_{0}^{+\infty} \int_{\mathbb{R}}\left|\theta_{t}(1)(x)\right|^{2}\left|P_{t} g(x)\right|^{2} \frac{d x d t}{t} \leq C\|g\|_{2}^{2},
$$

and by Carleson's lemma this is equivalent to (7).

Step 3: checking the Carleson measure estimate. This part of the argument is heavily specific to dimension one. Following Semmes [S], it starts with the observation that $\theta_{t}\left(\frac{1}{a}\right)=0$, while $\operatorname{Re} \frac{1}{a} \geq \delta$ a.e. for some $\delta>0$. Therefore, $\operatorname{Re} P_{t}\left(\frac{1}{a}\right) \geq \delta$, whence the simple but crucial inequality

$$
\left|\theta_{t}(1)(x)\right| \leq \frac{1}{\delta}\left|\theta_{t}(1)(x) P_{t}\left(\frac{1}{a}\right)(x)\right| .
$$

One then writes

$$
0=\theta_{t}\left(\frac{1}{a}\right)=\theta_{t}(1) P_{t}\left(\frac{1}{a}\right)+\tilde{\theta}_{t}\left(\frac{1}{a}\right),
$$

and by standard localisation arguments based on (4), inequality (8) implies

$$
\int_{0}^{|I|} \int_{I}\left|\tilde{\theta}_{t}\left(\frac{1}{a}\right)(x)\right|^{2} \frac{d x d t}{t} \leq C|I|
$$

The desired Carleson measure estimate immediately follows from this and (11). 


\section{The proof in any dimension: the Carleson measure crite- rion}

The proof in dimension $n$ is organised along the same steps. The first one remains unchanged until we arrive at the quadratic inequality

$$
\int_{0}^{+\infty}\left\|\left(I+t^{2} L\right)^{-1} t \operatorname{div} A \nabla f\right\|_{2}^{2} \frac{d t}{t} \leq C\|\nabla f\|_{2}^{2} .
$$

This time, as we will see, it is important to keep the term $\nabla f$, without replacing it by an arbitrary $L^{2}$ vector-valued function.

Denote again $\theta_{t}=\left(I+t^{2} L\right)^{-1} t \operatorname{div} A$, which is now an operator mapping vectorvalued functions to scalar functions. The second step is devoted to the

Lemma 3. The quadratic inequality (12) is implied by the Carleson measure estimate

$$
\int_{0}^{\ell_{Q}} \int_{Q}\left|\theta_{t}(I)(x)\right|^{2} \frac{d x d t}{t} \leq C|Q|
$$

where $Q$ is any cube with sides parallel to the axes, measure $|Q|$ and sidelength $\ell_{Q}$, and $\theta_{t}(I)$ denotes the vector-valued function $\left(\theta_{t}\left(e_{1}\right), \cdots, \theta_{t}\left(e_{n}\right)\right)$.

Only the stated implication is needed, though one may prove the full equivalence between the two inequalities: see [AT].

As in the one-dimensional case, Lemma 3 relies on the comparison between $\theta_{t}$ and $\theta_{t}(I) \cdot P_{t}$, applied to gradients of scalar functions:

$$
\int_{0}^{+\infty}\left\|\theta_{t} \nabla f-\theta_{t}(I) \cdot P_{t} \nabla f\right\|_{2}^{2} \frac{d t}{t} \leq C\|\nabla f\|_{2}^{2}
$$

where $P_{t}$ acts coordinatewise.

Here, Christ and Journé theorem is no more available, for the pointwise size estimates on $\theta_{t}(x, y)$ are no longer valid - but there is a substitute - , and there is no more regularity property with respect to $y$.

Let us be more detailed and begin with a discussion of size estimates. We have

$$
\theta_{t}(x, y)=-t A(y) \nabla_{y} R_{t}(x, y)
$$

where $R_{t}(x, y)$ is the kernel of the resolvent operator $\left(I+t^{2} L\right)^{-1}$. The best possible case is that of $A$ being real-valued, for then $R_{t}(x, y)$ fulfills pointwise estimates like

$$
\left|R_{t}(x, y)\right| \leq C t^{-2}|x-y|^{-n+2} e^{-\alpha \frac{|x-y|}{t}} .
$$

From this one can deduce weighted integral size estimates for $\theta_{t}(x, y)$ of the type

$$
\int_{\mathbb{R}^{n}}\left|\theta_{t}(x, y)\right| e^{\alpha^{\prime} \frac{|x-y|}{t}} d y \leq C .
$$

However, in the general complex-valued case, inequality (15) may fail, in quite a strong way: 
Theorem 4 ([ACT]). When $n \geq 5$, there exists an operator $L$ such that, for all $t>0,\left(I+t^{2} L\right)^{-1}$ is not bounded on $L^{\infty}$.

The adequate substitute to (15) is then the following:

Lemma 5. Let $E, E^{\prime}$ be two closed subsets of $\mathbb{R}^{n}$ with $d\left(E, E^{\prime}\right)>0$, and $F \in$ $L^{2}\left(\mathbb{R}^{n} ; \mathbb{C}^{n}\right)$ with $\operatorname{Supp} F \subset E^{\prime}$. Then

$$
\int_{E}\left|\theta_{t} F(x)\right|^{2} d x \leq C e^{-\alpha \frac{d\left(E, E^{\prime}\right)}{t}} \int_{E^{\prime}}|F(x)|^{2} d x
$$

for each $t>0$, with $C$ and $\alpha$ only depending on $\lambda$ and $\Lambda$.

This result, obtained from the Caccioppoli inequality, is the size estimate on the kernel of $\theta_{t}$ to be used constantly.

Regarding the regularity property of $\theta_{t}(x, y)$, a look at (14) shows that we cannot expect any hölderian behaviour with respect to $y$, even in a weak integral sense, unless the matrix $A$ has additional regularity. Nevertheless, the formula

$$
\left(I+t^{2} L\right)^{-1} t L=\frac{1}{t} I-\frac{1}{t}\left(I+t^{2} L\right)^{-1}
$$

implies that the divergence $\operatorname{div}_{y} \theta_{t}(x, y)$ behaves like $\frac{1}{t} R_{t}(x, y)$ when $x \neq y$. This is a key cancellation property of $\theta_{t}$, which explains the importance of keeping in (13) the gradient $\nabla f$. The same inequality with $F$ instead of $\nabla f$ is also true, but proving it is equivalent to proving $(\mathrm{K})$.

The proof of (13) starts with the decomposition

$$
\theta_{t}-\theta_{t}(I) \cdot P_{t}=\theta_{t}\left(I-P_{t}\right)+\left(\theta_{t}-\theta_{t}(I)\right) P_{t} .
$$

The first term in the right hand side leads to

$$
\theta_{t}\left(I-P_{t}\right) \nabla f=\theta_{t} \nabla\left(I-P_{t}\right) f .
$$

Using (17) we obtain

$$
\left\|\theta_{t}\left(I-P_{t}\right) \nabla f\right\|_{2} \leq C \frac{1}{t}\left\|\left(I-P_{t}\right) f\right\|_{2},
$$

and Plancherel theorem gives

$$
\int_{0}^{+\infty}\left\|\left(I-P_{t}\right) f\right\|_{2}^{2} \frac{d t}{t^{3}} \leq C\|\nabla f\|_{2}^{2}
$$

To handle the second term, we set $U_{t}=\left(\theta_{t}-\theta_{t}(I)\right) P_{t}$ and prove, using Lemma 5 , that the kernel of $U_{t}^{*} U_{t}$ satisfies size and regularity properties similar to (4) and (5), in their multidimensional versions. Since by construction $U_{t}(I)=0$, we conclude as in the proof of (8). Note that we use here the right regularisation induced by the multiplication by $P_{t}$, and the left regularisation induced by the $U_{t}^{*} U_{t}$ trick.

Once (13) is proved, Lemma 3 is obtained by appealing to Carleson's lemma as in dimension one. 


\section{The Carleson measure estimate in any dimension}

We thus have to prove the inequality

$$
\int_{0}^{\ell_{Q}} \int_{Q}\left|\theta_{t}(I)(x)\right|^{2} \frac{d x d t}{t} \leq C|Q|
$$

for all cubes $Q$.

Select a cube $Q$ and a direction $w$, i.e. a unit vector in $\mathbb{C}^{n}$. The main lemma is the following:

Lemma 6. There exists $\eta>0, M>0$ only depending on $n, \lambda$ and $\Lambda$, and a function $f_{Q, w} \in H_{l o c}^{1}\left(\mathbb{R}^{n}\right)$ such that

(i)

$$
\int_{5 Q}\left|\nabla f_{Q, w}\right|^{2} \leq M|Q|
$$

(ii) $f_{Q, w}$ is in the domain of $L$ over $5 Q$, in the sense that $L f_{Q, w}$ is square-integrable over $5 Q$, with

$$
\int_{5 Q}\left|L f_{Q, w}\right|^{2} \leq M \ell_{Q}^{-2}|Q|
$$

(iii) the mean values of $\nabla f_{Q, w}$, at scales varying from 0 to $\ell_{Q}$, are oriented along $w^{*}$ (the complex conjugate of $w$ ) over a substantial part of the Carleson box $\left.\left.B_{Q}=\right] 0, \ell_{Q}\right] \times Q$ : there exists a partition of $B_{Q}$ of the form

$$
B_{Q}=\bigcup_{Q^{\prime}} B_{Q^{\prime}} \cup \bigcup_{Q^{\prime \prime}} R_{Q^{\prime \prime}}
$$

where $\left.\left.R_{Q^{\prime \prime}}=\right] \frac{1}{2} \ell_{Q^{\prime \prime}}, \ell_{Q^{\prime \prime}}\right] \times Q^{\prime \prime}$ is the upper half of the Carleson box over $Q^{\prime \prime}$, and

- the cubes $Q^{\prime}$ are mutually disjoint dyadic subcubes of $Q$, with

$$
\sum_{Q^{\prime}}\left|Q^{\prime}\right| \leq(1-\eta)|Q|
$$

- the cubes $Q^{\prime \prime}$ are those dyadic subcubes of $Q$ which are not contained in any $Q^{\prime}$, with

$$
\begin{gathered}
\operatorname{Re} \int_{Q^{\prime \prime}} \nabla f_{Q, w} \cdot w \geq \frac{3}{4}\left|Q^{\prime \prime}\right|, \\
\int_{Q^{\prime \prime}}\left|\nabla f_{Q, w}\right| \leq M\left|Q^{\prime \prime}\right| .
\end{gathered}
$$


Let us admit this statement for the moment, and show how we use it.

We start with defining an angular localisation: to each direction $w$ associate a cone $\Gamma_{w}$ of small aperture, and select a finite set $W$ of directions such that

$$
\mathbb{C}^{n}=\bigcup_{w \in W} \Gamma_{w}
$$

is a partition, up to negligible sets. Then write

$$
\begin{gathered}
\theta_{t}(I)=\sum_{w \in W} \theta_{t}(I) 1_{\theta_{t}(I) \in \Gamma_{w}} \\
\stackrel{\text { def }}{=} \sum_{w \in W} \gamma_{t, w}
\end{gathered}
$$

so that it suffices to prove a Carleson measure estimate for each $\gamma_{t, w}$.

Then we use Lemma 6 to obtain a comparison between two measures, in the spirit of the one-dimensional third step. We have indeed

$$
\iint_{B_{Q}}\left|\gamma_{t, w}\right|^{2} \frac{d x d t}{t}=\sum_{Q^{\prime \prime}} \iint_{R_{Q^{\prime \prime}}}\left|\gamma_{t, w}(x)\right|^{2} \frac{d x d t}{t}+\sum_{Q^{\prime}} \iint_{B_{Q^{\prime}}}\left|\gamma_{t, w}(x)\right|^{2} \frac{d x d t}{t}
$$

When $(t, x) \in R_{Q^{\prime \prime}}$, set

$$
\frac{1}{\left|Q^{\prime \prime}\right|} \int_{Q^{\prime \prime}} \nabla f_{Q, w}=S_{t}^{Q} \nabla f_{Q, w}(x)
$$

The operator $S_{t}^{Q}$ is the dyadic approximation of the identity associated to the cube $Q$.

By (19), (20) and the definition of $\gamma_{t, w}$ (with an appropriate choice of the aperture of $\left.\Gamma_{w}\right)$ we have

$$
\left|\gamma_{t, w}(x)\right| \leq 10\left|\gamma_{t, w}(x) \cdot S_{t}^{Q} \nabla f_{Q, w}(x)\right|
$$

pointwise. Therefore we get

$$
\begin{aligned}
\iint_{B_{Q}}\left|\gamma_{t, w}\right|^{2} \frac{d x d t}{t} \leq 100 \sum_{Q^{\prime \prime}} \iint_{R_{Q^{\prime \prime}}}\left|\gamma_{t, w}(x) \cdot S_{t}^{Q} \nabla f_{Q, w}(x)\right|^{2} \frac{d x d t}{t} & \\
& +||\left|\gamma_{t, w}\right|^{2} \frac{d x d t}{t} \|_{c} \sum_{Q^{\prime}}\left|Q^{\prime}\right|
\end{aligned}
$$

Here, $\|\mu\|_{c}$ denotes $\sup _{Q} \frac{1}{|Q|} \mu\left(B_{Q}\right)$.

We may assume that $\left\|\left|\gamma_{t, w}\right|^{2} \frac{d x d t}{t}\right\|_{c}$ is finite, thanks to a standard truncation over the variable $t$. Invoking (18), dividing by $Q$ and taking the supremum over $Q$, we obtain the following comparison

$$
\left\|\left|\gamma_{t, w}\right|^{2} \frac{d x d t}{t}\right\|_{c} \leq \frac{100}{\eta} \sup _{Q} \frac{1}{|Q|} \iint_{B_{Q}}\left|\gamma_{t, w}(x) \cdot S_{t}^{Q} \nabla f_{Q, w}(x)\right|^{2} \frac{d x d t}{t} .
$$

Now we are done: each integral in the right hand side is comparable to $\left\|\sqrt{L} f_{Q, w}\right\|_{L^{2}(5 Q)}^{2}$, as can be seen by taking the second step in reverse order, and 
suitably modifying it with localisation arguments. Since the function $f_{Q, w}$ is in the domain of $L$ over $5 Q$, the operator $\sqrt{L}$ acts nicely on it, and we get the desired control, using (i) and (ii) in Lemma 6.

It remains to prove this lemma. We choose

$$
f_{Q, w}=\left(I+\epsilon^{2} \ell_{Q}^{2} L\right)^{-1} \Phi_{Q} \cdot w^{*}
$$

where $\Phi_{Q}(x)=x-x_{Q}, x_{Q}$ being the center of $Q$. The real $\epsilon$ is a small parameter that will be chosen independently of $Q$ and $w$.

The estimates (i) and (ii) are proved by studying the commutator between the resolvent and the multiplication operator by a Lipschitz function. Another useful estimate is

$$
\int_{5 Q}\left|f_{Q, w}-\Phi_{Q} \cdot w^{*}\right|^{2} \leq C \epsilon^{2} \ell_{Q}^{2}|Q|
$$

With (i), this implies that $\nabla f_{Q, w}$ is in the mean close to $w^{*}$ :

$$
\left|\int_{Q} \nabla f_{Q, w}-w^{*}\right| \leq C \sqrt{\epsilon}|Q|
$$

This inequality is a direct consequence of the following result, applied to $g=f_{Q, w}-$ $\Phi_{Q} \cdot w^{*}$.

Lemma 7. If $g \in H^{1}(Q)$ then

$$
\left|\int_{Q} \nabla g\right| \leq C \ell_{Q}^{\frac{n-1}{2}}\left(\int_{Q}|g|^{2}\right)^{\frac{1}{4}}\left(\int_{Q}|\nabla g|^{2}\right)^{\frac{1}{4}}
$$

for some absolute constant $C$.

Inequality (22) is the cornerstone of the stopping-time argument defining the subcubes $Q^{\prime}$ and $Q^{\prime \prime}$. Let us say that $Q^{\prime \prime}$, a dyadic subcube of $Q$, is good when (19) and (20) hold true.

Up to choosing $\epsilon$ small enough, (22) shows us first that $Q$ is good. We subdivide it in $2^{n}$ subcubes and check whether each one is good or not. If not, we stop and label the bad subcube in question as a $Q^{\prime}$. If yes, we subdivide it and continue the process. This is a classical procedure, reminiscent of the Calderón-Zygmund decomposition.

We end up with a collection of bad dyadic subcubes $Q^{\prime}$ which are maximal with respect to the condition

$$
\operatorname{Re} \int_{Q^{\prime}} \nabla f_{Q, w} \cdot w<\frac{3}{4}\left|Q^{\prime}\right|
$$

or

$$
\int_{Q^{\prime}}\left|\nabla f_{Q, w}\right|>M\left|Q^{\prime}\right|
$$

The union of those cubes fulfilling the second part of the condition is a small subset of $Q$, provided $M$ is appropriately chosen. Now, if $Q^{\prime}$ satisfies the first part, we have

$$
\left|Q^{\prime}\right|<4 \operatorname{Re} \int_{Q^{\prime}} 1-\nabla f_{Q, w} \cdot w
$$


and therefore

$$
\sum\left|Q^{\prime}\right|<4\left|\operatorname{Re} \int_{\cup Q^{\prime}} 1-\nabla f_{Q, w} \cdot w\right|
$$

But inequality (22) prevents the set $\cup Q^{\prime}$ from being too big in $Q$. When quantifying precisely this, we arrive at

$$
\sum\left|Q^{\prime}\right| \leq(1-\sqrt{\epsilon}+o(\sqrt{\epsilon}))|Q|
$$

This leads to fixing $\epsilon$, and concludes the proof of the main lemma (it is indeed obvious that the good subcubes $Q^{\prime \prime}$ do satisfy the required conditions).

The reader will have understood that the aim of these notes is to introduce the ideas of the proof, and - hopefully — to help the lecture of [AHLMT], where all the omitted arguments may be found.

\section{References}

[ACT] Auscher, P., Coulhon, T., Tchamitchian, P. Absence de principe du maximum pour certaines équations paraboliques complexes, Coll. Math., 171 (1996), 87-95.

[Ahlt] Auscher, P., Hofmann, S., Lewis, J., Tchamitchian, P. Extrapolation of Carleson measures and the analyticity of Kato's square root operator, Acta Math., to appear.

[Ahlmt Auscher, P., Hofmann, S., Lacey, M., Mcintosh, A., TChamitchian, P. The solution of the Kato square root problem for second order elliptic operators on $\mathbb{R}^{n}$, submitted.

[Ahllmt] Auscher, P., Hofmann, S., Lacey, M., Lewis, J., Mcintosh, A., Tchamitchian, P. La solution des conjectures de Kato, $C$. $R$. Acad. Sci. Paris, 327, Série I (2001).

[AMT $]$ Auscher, P., Mcintosh, A., Tchamitchian, P. Heat kernel of complex elliptic operators and applications, J. Funct. Anal., 152 (1998), 22-73.

[AT95] Auscher, P., Tchamitchian, P. Calcul fonctionnel précisé pour des opérateurs elliptiques complexes en dimension un (et applications à certaines équations elliptiques complexes en dimension deux), Ann. Inst. Fourier, 45 (1995), 721-778.

[AT] Auscher, P., Tchamitchian, P. Square root problem for divergence operators and related topics, Astérisque 249, Société Mathématique de France, 1998.

[C] Calderón, A. P. Commutators of singular integral operators, Proc. Nat. Acad. Sci. U.S.A. 53 (1965), 1092-1099. 
[CDM] Coifman, R., Deng, D., Meyer, Y. Domaine de la racine carrée de certains opérateurs différentiels accrétifs, Ann. Inst. Fourier $\mathbf{3 3}$ (1983), 123-134.

[CJ] Christ, M., Journé, J.-L. Polynomial growth estimates for multilinear singular integral operators, Acta Math. 159 (1987), 51-80.

[CMM] Coifman, R., M'Intosh, A., Meyer, Y. L'intégrale de Cauchy définit un opérateur borné sur $L^{2}(\mathbb{R})$ pour les courbes lipschitziennes, Ann. Math. 116 (1982), 361-387.

[FJK $]$ FABes, E., Jerison, D., Kenig, C. Multilinear square functions and partial differential equations, Amer. J. of Math. 107 (1985), $1325-1367$.

[J] Journé, J.-L. Remarks on the square root problem, Pub. Math. 35 (1991), 299-321.

[K] Kato, T. Fractional powers of dissipative operators, J. Math. Soc. Japan 13 (1961), 246-274.

[KM] Kenig, C., Meyer, Y. The Cauchy integral on Lipschitz curves and the square root of second order accretive operators are the same, Recent Progress in Fourier Analysis (I. Peral, ed.), Math. Studies 111, North Holland, 1985, 123-145.

[M72] M Intosh, A. On the Comparability of $A^{1 / 2}$ and $A^{* 1 / 2}$, Proc. Amer. Math. Soc. 32 (1972), 430-434.

[M82] M Intosh, A. On representing closed accretive sesquilinear forms as $\left(A^{1 / 2} u, A^{* 1 / 2} v\right)$, Collège de France Seminar, Volume III (H. Brezis and J.-L. Lions, eds.), Research Notes in Mathematics 70, Pitman, 1982, 252-267.

[M83] M'Intosh, A. Square roots of operators and applications to hyperbolic PDE, Miniconference on Operator Theory and Partial Differential Equations (Canberra), Center for Math. and Appl., The Australian National University, 1983.

[M85] M M Intosh, A. Square roots of elliptic operators, J. Funct. Anal. 61 (1985),307-327.

[M86] McIntosh, A. Operators which have an $H^{\infty}$ functional calculus, Miniconference on Operator Theory and Partial Differential Equations (Canberra), Center for Math. and Appl., The Australian National University, 1986.

[S] Semmes, S. Square function estimates and the T(b) Theorem, Proc. Amer. Math. Soc. 110 (1990), 3, 721-726. 
[Y] Yagi, A. Coïncidence entre des espaces d'interpolation et des domaines de puissances fractionnaires d'opérateurs, C. R. Acad. Sci. Paris 299, Série I (1984), 173-176.

Faculté des Sciences et Techniques de St.-JÉrôme

Université D'AiX-MARSEILLE III

Avenue Escadrille Normandie-Niemen

F-13397 Marseille Cedex 20

tchamphi@math.u-3mrs.fr 